\title{
Adesão a um Programa de Triagem Auditiva Neonatal
}

\section{Adherence to a Neonatal Hearing Screening Program}

\author{
Maria de Fátima de Campos Françozo \\ Assistente Social. Doutora em Saúde Mental. Professora do Centro \\ de Estudos e Pesquisas em Reabilitação "Prof. Dr. Gabriel Porto"/ \\ Faculdade de Ciências Médicas da Unicamp. \\ Endereço: Rua Tessália Vieira de Camargo, 126, CEP 13084-971, \\ Campinas, SP, Brasil. \\ E-mail: $m f c f \bowtie f c m$.unicamp.br; m. fatimafœgmail.com

\section{Gabriela Abrahão Masson} \\ Assistente social. Mestranda em Serviço Social. Universidade Esta- \\ dual Paulista "Júlio de Mesquita Filho"/Campus de Franca. \\ Endereço: Av. Eufrásia Monteiro Petráglia, 900, CEP 14409-160, \\ Franca, SP, Brasil. \\ E-mail: gabrielaabrahomasson®yahoo.com.br

\section{Tereza Ribeiro de Freitas Rossi} \\ Fonoaudióloga. Doutora em Educação Física Adaptada; Professora \\ do Centro de Estudos e Pesquisas em Reabilitação "Prof. Dr. Gabriel \\ Porto"/Faculdade de Ciências Médicas da Unicamp. \\ Endereço: Rua Tessália Vieira de Camargo, 126, CEP 13084-971, \\ Campinas, SP, Brasil. \\ E-mail: terezafrøfcm.unicamp.br
}

\section{Maria Cecília Marconi Pinheiro Lima}

Fonoaudióloga. Doutora em Ciências Médicas: área de neurologia. Professora do Centro de Estudos e Pesquisas em Reabilitação "Prof. Dr. Gabriel Porto"/Faculdade de Ciências Médicas da Unicamp. Endereço: Rua Tessália Vieira de Camargo, 126, CEP 13084-971, Campinas, SP, Brasil.

E-mail: ceclimaœfcm.unicamp.br

\section{Maria Francisca Colella dos Santos}

Fonoaudióloga. Doutora em Ciências dos Distúrbios da Comunicação Humana pela UNIFESP. Professora do Centro de Estudos e Pesquisas em Reabilitação "Prof. Dr. Gabriel Porto"/Faculdade de Ciências Médicas da Unicamp.

Endereço: Rua Tessália Vieira de Camargo, 126, CEP 13084-971, Campinas, SP, Brasil.

E-mail: mfcolellaðfcm.unicamp.br

Fontes de auxílio: FUNDAP - Fundação para o Desenvolvimento

- SP (bolsa de aprimoramento profissional).

\section{Resumo}

A Triagem Auditiva Neonatal tem se efetivado mundialmente como meio para detecção precoce da surdez. Na Unicamp, desde 2002, os recém-nascidos na maternidade do Centro de Atenção Integral à Saúde da Mulher são agendados para a triagem auditiva no Centro de Estudos e Pesquisas em Reabilitação "Prof. Dr. Gabriel Porto". No entanto, nem todos vêm para a triagem e alguns abandonam o processo de avaliação antes do diagnóstico. O objetivo desta pesquisa foi caracterizar as taxas de adesão de lactentes ao Programa de Triagem Auditiva Neonatal. Tratou-se de pesquisa que utilizou dados contidos nos prontuários dos lactentes que efetuaram a triagem no período de fevereiro a novembro de 2007. Permaneceram no alojamento conjunto do CAISM 2107 lactentes e vieram para a triagem 1310. Dentre aqueles que não passaram na triagem (92 lactentes), realizaram o exame de PEATE-A 73 lactentes. A adesão na primeira etapa da triagem foi de $62,17 \%$, e na segunda, 79,34\%. As taxas de adesão são inferiores às preconizadas pelo Joint Comittee on Infant Hearing e encontradas em alguns países desenvolvidos. No entanto, aproximam-se de outras experiências brasileiras de programas de triagem auditiva neonatal. 0 acompanhamento sistemático às famílias dos lactentes que não passaram na primeira avaliação e a conscientização destas sobre a detecção precoce da perda auditiva e suas consequências podem ter contribuído para o aumento da taxa de adesão na segunda etapa da triagem.

Palavras-chave: Triagem neonatal; Lactente; Perda auditiva; Adesão. 


\section{Abstract}

Neonatal hearing screening programs have been developed worldwide. Since 2002, the newborns from the Center for Integral Assistance to Women's Health at UNICAMP have been referred to hearing screening at the Center for Studies and Research on Rehabilitation "Prof. Dr. Gabriel Porto". Nevertheless, not all newborns come to the screening and others drop out before the procedures are completed. The goal of this research was to characterize the newborns' adherence rates to the Neonatal Hearing Screening Program. A retrospective investigation was carried out, collecting data on the records of all cases screened from February to November of 2007. A total of 2107 infants were referred to hearing screening and 1310 actually came. Among those who failed the test (92 infants), 73 returned for the retest with the use of BAEP. The adherence rate in the first stage of the screening was $62.17 \%$ and, in the second, 79.34\%. These rates are bellow the ones recommended by the JCIH as well as bellow those reached by hearing screening programs of some developed countries. However, they are similar to other Brazilian experiences of neonatal hearing screening programs. A close follow up of the families of those infants who failed the first stage of the screening, as well as the orientation given to them about early detection of hearing loss and its consequences, may have contributed to the increase in the adherence rate in the second stage of the screening.

Keywords: Neonatal screening; Infant; Hearing loss; Adherence.

\section{Introdução}

A audição constitui-se em um pré-requisito para a aquisição e o desenvolvimento da linguagem (Gatto e Tochetto, 2007). Assim, a deficiência auditiva nos primeiros anos de vida de uma criança interfere na aquisição e no desenvolvimento da fala e da linguagem.

Estudos realizados por Yoshinaga-Itano e colaboradores (1998) indicam que quando a perda auditiva é diagnosticada até os três meses de vida e a intervenção terapêutica é iniciada até os seis meses, o desenvolvimento da compreensão e da expressão da linguagem, bem como o relacionamento social e o desempenho acadêmico da criança com perda auditiva, podem ser compatíveis com os de crianças ouvintes da mesma faixa etária.

A incidência de perda auditiva bilateral significante em neonatos saudáveis é estimada entre $1 \mathrm{a}$ 3 neonatos em cada 1000 nascimentos e em cerca de 2 a $4 \%$ nos provenientes de Unidades de Terapia Intensiva (Comitê Brasileiro sobre Perdas Auditivas na Infância, 200o).

Segundo Olusanya e colaboradores (2004), a cada ano, aproximadamente 126.000 a 500.000 crianças nascem com significativa perda auditiva e 9o\% destas se encontram em países em desenvolvimento. Os mesmos autores ainda apontam que, em 1995, a Organização Mundial da Saúde estimou que dos 78 milhões de pessoas que suspeitavam ter uma deficiência auditiva maior que $40 \mathrm{~dB}, 8$ milhões tinham até 18 anos e moravam em países em desenvolvimento.

A detecção precoce da perda auditiva não é apenas o primeiro, mas também o mais importante passo para a obtenção de bons resultados no desenvolvimento social e educacional em crianças com perda auditiva (Ozcebe e col., 2005). As Academias Americanas de Audiologia, Otorrinolaringologia e Pediatria reunidas no Joint Committee on Infant Hearing (JCIH, 2007) recomendam a triagem auditiva neonatal universal antes dos três meses de idade da criança e o início da intervenção antes dos seis meses de idade.

A Triagem Auditiva Neonatal Universal (TANU) constitui-se como um processo inicial de avaliação da audição infantil que permite a detecção precoce de possíveis alterações auditivas, abrangendo todos 
os neonatos, inclusive aqueles que não possuem indicadores de risco para a surdez (JCIH, 2007).

A introdução de programas de triagem auditiva neonatal em países em desenvolvimento ainda é considerada inacessível, por causa de uma série de razões, dentre elas, os custos associados à triagem e aos serviços de seguimento (Olusanya e col., 2004).

Os primeiros programas de triagem auditiva neonatal neste país iniciaram-se em 1987 e, no ano seguinte, ocorreram as primeiras iniciativas para divulgação da TAN, com a criação do Grupo de Apoio à Triagem Auditiva Neonatal (GATANU). A primeira recomendação nacional para a implantação da triagem foi elaborada em 1999 pelo Comitê Brasileiro sobre Perdas Auditivas na Infância (Comitê Brasileiro sobre Perdas Auditivas na Infância, 200o).

Atualmente, a Política Nacional de Atenção à Saúde Auditiva (Brasil, 2004) prevê o atendimento integral a pessoas com deficiência auditiva por meio de ações que englobam a atenção básica (trabalhos de promoção da saúde, prevenção e identificação precoce de problemas auditivos), de média complexidade (realizar triagem e monitoramento da audição) e de alta complexidade (realizar atenção diagnóstica e terapêutica especializada), com o fornecimento de prótese auditiva e terapia fonoaudiológica.

No município de Campinas, o Decreto $\mathrm{n}^{0}$.14.640, de 16/02/2004, regulamenta a Lei $n^{0} .10 .759$ que dispõe da obrigatoriedade da realização de exames que detectem a surdez ou alterações correlatas nas maternidades e estabelecimentos hospitalares. (Campinas, 2004).

Na Universidade Estadual de Campinas (Unicamp), a TAN tem sua gênese no Setor de Neonatologia do Departamento de Pediatria da Faculdade de Ciências Médicas/CAISM, que adotou o programa de triagem auditiva neonatal, realizado antes da alta hospitalar, inicialmente avaliando os recémnascidos que necessitavam de internação na UTI Neonatal ou Unidade de Cuidados Intermediários (Lima, 2004).

O projeto "Detecção de Alterações Audiológicas em Neonatos (DAANE)", criado em 2002, ampliou a triagem a neonatos de alojamento conjunto. Desenvolvido por profissionais do Centro de Estudos e Pesquisas em Reabilitação "Prof. Dr. Gabriel Porto" (CEPRE) em parceria com profissionais do CAISM, o
Programa de TAN passou por modificações ao longo dos anos, buscando-se encontrar a melhor maneira de realizar a avaliação. Atualmente, a triagem é realizada no CEPRE, local para o qual os lactentes são encaminhados, tendo a data da avaliação agendada quando da alta hospitalar. A avaliação é realizada no primeiro mês de vida da criança.

Ao chegar para a triagem no CEPRE, antes da realização do exame audiológico, as mães e acompanhantes dos lactentes participam de um grupo de orientação sobre a TAN sob a responsabilidade de fonoaudiólogas e assistentes sociais. A seguir, o lactente é avaliado por uma fonoaudióloga por meio do método fisiológico de emissões otoacústicas transientes (EOAT) e é realizado o levantamento dos indicadores de risco para surdez, segundo o que o Joint Committee on Infant Hearing (2000) sugere.

Nos casos em que a criança falha no teste, a mãe é orientada e encaminhada ao CAISM onde é aplicado o Potencial Evocado Auditivo de Tronco Encefálico, modo automático (PEATE-A). Em caso de falha no reteste, a mãe é orientada sobre os procedimentos seguintes e, principalmente, é acolhida tendo em vista sua preocupação com o resultado do teste. Enfatizase, nesse momento, a importância da continuidade da avaliação, solicitando que traga o resultado dos procedimentos de diagnóstico audiológico e otorrinolaringológico à equipe de profissionais do Cepre, para que seja dado seguimento à reabilitação.

O Serviço Social, em alguns casos, mediante estudo social, disponibiliza passagens de ônibus para o retorno da mãe, ou verifica com ela outras situações que possam dificultar seu retorno. Nos casos em que a criança faltou à avaliação agendada ou que realizou o exame, mas não voltou ao Cepre, mais uma vez, a assistente social entra em contato com os responsáveis do lactente para verificar motivos que levaram ao não comparecimento, iniciando assim um processo que visa garantir a finalização dos procedimentos.

Apesar do acompanhamento sistemático dos lactentes que não passam na avaliação inicial, há aqueles que não retornam, não completando o processo de avaliação.

Em relação ao funcionamento da TAN na Unicamp, há poucos estudos sobre as taxas de adesão dos lactentes (Françozo e col., 2007; Rossi e col., 2003) e nenhum relacionado ao modelo atual de 
funcionamento do Programa. Entende-se por adesão o comparecimento do lactente para realização da triagem. Como há aqueles lactentes que falham na triagem auditiva e devem realizar um reteste por meio do exame de PEATE-A, considerou-se que essa etapa também deveria ser avaliada em termos da participação do lactente. Assim, a adesão à triagem deveria ser verificada em duas etapas: a primeira, daqueles que, sendo encaminhados, vieram efetivamente para a avaliação, e a segunda, aqueles casos em que o lactente "não passou" e realizou a segunda avaliação (reteste) por meio do PEATE-A.

Dessa forma, o objetivo deste estudo foi caracterizar as taxas de adesão de lactentes ao Programa de Triagem Auditiva Neonatal.

\section{Métodos}

Tratou-se de pesquisa realizada em prontuários de todos os lactentes que compareceram à TAN no período de fevereiro a novembro de 2007.

Foram efetuados os seguintes procedimentos em relação à triagem propriamente: ao trazer o lactente para a triagem, as mães e acompanhantes participavam de um grupo de orientação sobre a TAN sob a responsabilidade de fonoaudiólogas e assistentes sociais. A seguir, o lactente era avaliado por uma fonoaudióloga por meio do método fisiológico de emissões otoacústicas transientes (EOAT) e era realizado o levantamento dos indicadores de risco para surdez, segundo o que o Joint Committee on Infant Hearing (200o) sugere. As emissões otoacústicas são sons gerados pelas células ciliadas externas da cóclea, captados no meato acústico externo. $\mathrm{O}$ teste foi realizado por meio do equipamento Echocheck-Otodynamics, equipamento automático que mostra que o neonato passou no teste quando apresenta nível de relação sinal/ruído acima de 6 dB (segundo manual do equipamento Echocheck versão 3), resposta observada quando a luz verde do equipamento se acende.

No caso de o neonato apresentar falha nas emissões otoacústicas transientes, foi encaminhado para reteste, realizado no CAISM, por meio do Potencial Evocado Auditivo de Tronco Encefálico AutomáticoPEATE-A. O equipamento utilizado foi o da NATUS, Algo 2e, que possibilita pesquisar resposta para estímulo do tipo clique na intensidade de $35 \mathrm{~dB}, 40$ e $70 \mathrm{~dB}$. Para cliques de $35 \mathrm{~dB}$, o equipamento de triagem ALGO gerou um resultado PASSA quando coletou dados suficientes para estabelecer com confiança estatística de $99,96 \%$ que um sinal de potencial evocado de tronco encefálico estava presente e consistente com o padrão de normalidade, para no mínimo 100 o varreduras. Considerou-se que o lactente FALHA quando não apresenta resposta para $35 \mathrm{~dB}$ em pelo menos uma orelha. Nesse caso, a testagem prosseguiu com a pesquisa das respostas do lactente para as intensidades de 40 e $70 \mathrm{~dB}$. Durante a aplicação dos testes, os neonatos ficaram posicionados no colo da mãe ou do pai, em estado tranquilo ou sono natural.

Em relação aos procedimentos de coleta de dados dos prontuários, estes foram obtidos por meio de documentos utilizados em atendimentos com os usuários. Foram colhidas informações dos documentos: Livro de registro dos nascimentos, extraindo-se dados relativos ao número de crianças que permaneceram no alojamento conjunto; Folha de estudo social, extraindo-se as informações relativas ao número de crianças que vieram para a triagem e o número daquelas que não passaram no teste; Folha de agendamento para o PEATE-A, com informações relativas ao número de crianças encaminhadas, o motivo do encaminhamento para o exame e o número de crianças que retornaram, com o referido resultado.

A presente pesquisa foi aprovada pelo Comitê de Ética em Pesquisa da Faculdade de Ciências Médicas da Unicamp - Parecer 558/2007.

\section{Resultados}

Para verificar a taxa de adesão dos lactentes na primeira etapa da triagem auditiva, foi feito um levantamento dos neonatos que nasceram e permaneceram no alojamento conjunto do CAISM entre os meses de fevereiro e novembro de 2007 e que compareceram ao Cepre para a triagem entre os meses de março e dezembro de 2007. 0 agendamento para a triagem é feito no primeiro mês de vida da criança, por isso a diferença nos meses de nascimento e agendamento.

Conforme mostra a Tabela 1, foram encaminhados pelo CAISM 2107 neonatos e 1310 compareceram para a triagem. A taxa de adesão nessa primeira etapa atingiu $62,17 \%$. 
Tabela I - Neonatos do alojamento conjunto do CAISM segundo o comparecimento para a TAN ( $\left.\mathrm{N}^{\circ} \mathrm{e} \%\right)$

\begin{tabular}{lcc} 
Comparecimento & N & $\%$ \\
\hline Compareceram para a triagem & 1310 & $62,17 \%$ \\
Não compareceram para a triagem & 797 & $37,83 \%$ \\
Total & 2107 & $100 \%$ \\
\hline
\end{tabular}

Para identificar a taxa de retorno na segunda etapa do processo de triagem foi levantado o número de lactentes efetivamente triados no período de março e dezembro de 2007, isto é, 1214 lactentes ( 96 lactentes não efetuaram a triagem por diferentes razões: comparecerem em horário ou dia não agendados e em que o serviço de triagem não funciona na instituição; pelo fato de a criança estar muito resfriada ou ainda por estar o aparelho em conserto). O número de neonatos que passou na avaliação inicial do CEPRE foi 1122 (92,42\%), sendo que 92 (7,57\%) lactentes não passaram (Tabela 2). Realizaram o exame PEATE-A 73 lactentes. A taxa de adesão nessa segunda etapa (reteste) foi de $79,34 \%$.

Tabela 2 - Neonatos que falharam nas EOAT e foram encaminhados ao PEATE-A ( ${ }^{\circ}$ e \%)

\begin{tabular}{lcc} 
Presença no exame & $N$ & $\%$ \\
Realizaram o exame & 73 & 79,34 \\
Faltaram & 19 & 20,65 \\
Total & 92 & $100 \%$ \\
\hline
\end{tabular}

Dentre os lactentes que realizaram o exame de PEATE-A, passaram 53 neonatos (57,60\%), falharam necessitando de acompanhamento 17 neonatos $(23,28)$ e em 3 neonatos $(3,26 \%)$ foi impossível a conclusão do exame, com necessidade de outros procedimentos audiológicos.

Se somarmos o número de lactentes que passou na triagem inicial (1122) ao número de lactentes que passou no reteste (53), teremos um total de 1175 lactentes que passaram na triagem, ou seja, $96,78 \%$ dos casos.

Em relação à resposta ao PEATE-A, verificou-se 8 lactentes com falha unilateral de $35 \mathrm{~dB}$ e $4 \mathrm{O} \mathrm{dB}$, falha bilateral em $35 \mathrm{~dB}$ e $40 \mathrm{~dB}$ foi detectada em 6 lactentes; falha unilateral em $70 \mathrm{~dB}$ em 2 lactentes e, em 1 lactente, foi detectada falha em $70 \mathrm{~dB}$ bilateralmente (Tabela 3).

\begin{tabular}{|c|c|c|}
\hline Resultados PEATE-A & $\mathrm{N}$ & $\%$ \\
\hline Passaram & 53 & $72,60 \%$ \\
\hline Falha Bilateral 35 e $40 \mathrm{~dB}$ & 6 & $8,21 \%$ \\
\hline Falha Bilateral $70 \mathrm{~dB}$ & I & $1,36 \%$ \\
\hline Falha Unilateral 35 e $40 \mathrm{~dB}$ & 8 & $10,95 \%$ \\
\hline Falha Unilateral $70 \mathrm{~dB}$ & 2 & $2,73 \%$ \\
\hline Impossivel concluir & 3 & $4,10 \%$ \\
\hline Total & 73 & $100 \%$ \\
\hline
\end{tabular}

\section{Discussão}

O Joint Committee on Infant Hearing (2007) estabeleceu índices de qualidade para programas de Triagem Auditva Neonatal, recomendando que a porcentagem de neonatos que devem completar a triagem no primeiro mês de idade deve ser maior que $95 \%$, ou seja, valor muito acima daquele obtido em nosso estudo (62,17\%). No entanto, comparando-se a taxa do estudo atual àquela encontrada por Fernandes (2005), no período inicial de implantação do Programa de Triagem Auditiva Neonatal na Unicamp, que foi de 52,3\%, verifica-se um aumento significativo de participação dos lactentes.

Podemos atribuir esse fato à maior divulgação sobre a TAN, seja por meio das legislações que a ratificam e que, indiretamente, são resultado de uma mobilização maior da população, seja pela divulgação do exame por alguns meios de comunicação, como, por exemplo, outdoors espalhados pela cidade de Campinas, seja ainda pelo tempo de implantação do próprio programa na Unicamp. Dessa forma, a TAN começa a ser conhecida e incorporada pela população, tornando-se mais um dos exames pósnatais de rotina.

Dados da literatura internacional, no entanto, indicam taxas maiores de adesão. De acordo com estudo realizado em nove hospitais do Texas, entre os anos de 1994 a 1997, Finitzo e colaboradores (1998) encontraram $96,8 \%$ de lactentes triados. 0 protocolo observado implicava na admissão da triagem e de- 
tecção realizada no quarto, antes da alta hospitalar. Podemos supor que a realização do exame neste momento ainda se constitui como um fator decisivo na efetividade de Programas de TANU.

Estudo sobre a triagem auditiva neonatal universal realizada em maternidades de Massachusetts, USA, no período de 2002-2003, identificou uma taxa de $99 \%$ de lactentes triados naquele estado (Liu e col., 2008).

Uma pesquisa que estudou a efetividade da primeira fase de implantação do Programa Nacional de Triagem Auditiva Neonatal na Inglaterra e que cobriu 170.000 nascimentos/ano indicou que foram triados $96 \%$ dos lactentes (Uus e Bamford, 2006). Outro estudo, este realizado na região de Siena - Itália, levantou dados relativos à triagem auditiva neonatal no período de o1 de abril de 1998 a 31 de julho de 2006. Dentre 21.125 nascidos vivos, foram testados 19.700 neonatos, ou seja, perderam a triagem de rotina $6,74 \%$ dos casos (De Capua e col., 2007).

No Brasil, estudo recente foi realizado por Griz e colaboradores (2009) na cidade de Recife (PE), em três hospitais públicos. Foram convidadas a participar do programa de triagem auditiva neonatal 1035 mães cujos neonatos nasceram em um dos hospitais. Elas foram entrevistadas para que se identificasse alguns aspectos da situação socioeconômica e demográfica e para que fosse esclarecida a triagem auditiva. Foi então efetuado agendamento para a triagem do lactente após um mês da alta hospitalar, com triagem opcional. Apenas 149 mães retornaram para participar da triagem (14,3\%).

Em relação à porcentagem daqueles lactentes que passaram na triagem, verificamos que a taxa encontrada em nosso estudo (96,78\%) está dentro daquela estabelecida pelo JCIH (2007), que considera que deve ser menos de $4 \%$ o número de encaminhamentos para avaliação diagnóstica compreensiva dentre todos os recém-nascidos que falham na triagem inicial e nos retestes subsequentes antes da avaliação audiológica compreensiva completa.

Porcentagens abaixo daquelas identificadas em nosso estudo, entretanto, podem ser encontradas na literatura internacional, como, por exemplo, no Colorado, que atingiu 93,51\% dos casos (Mehl e Thomson, 1998). O estudo realizado em Siena, na Itália (De Capua e col., 2007), encaminhou para avaliação com
PEATE 1549 lactentes, ou seja, 8,53\% dos casos triados. No Texas, o estudo de Finitzo e colaboradores (1998) identificou que passaram na triagem $96,6 \%$ dos neonatos, mas no protocolo seguido, em alguns momentos desta triagem inicial, foram utilizadas duas tecnologias para a realização da triagem, como as emissões e o PEATE-A.

0 estudo relativo à triagem no estado de Massachusetts (Liu e col., 2008) encontrou apenas $1 \%$ de lactentes que não passou no teste, enquanto que o estudo realizado na Inglaterra (Uus e Bamford, 2006) identificou que 3\% dos lactentes não passaram na triagem inicial.

Quando verificamos a adesão à segunda etapa do processo de triagem (reteste), isto é, quando verificamos também o retorno daqueles que falharam na triagem e que completaram o exame de PEATE, identificamos a taxa de $79,34 \%$, ou seja, $20,66 \%$ dos casos não retornaram.

$\mathrm{Na}$ literatura internacional, foram encontradas diferentes taxas relativas ao não retorno para a segunda etapa da triagem: $31,5 \%$ no estudo de Finitzo e colaboradores (1998) e $25 \%$ em pesquisa realizada por Isaacson (2000).

No estudo realizado em Massachusetts e mencionado anteriormente (Liu e col., 2008), 11\% das crianças que não passaram na triagem auditiva não usaram serviços de avaliação audiológica, ou seja, não retornaram para seguimento. Na Inglaterra, os resultados do estudo realizado por Uus e Bamford (2006) identificaram que foram perdidos no seguimento aproximadamente $10 \%$ dos casos. E o estudo conduzido por De Capua e colaboradores (2007), na Itália, apontou perda de 255 casos, isto é, $16,46 \%$ dos lactentes não retornaram para completar a avaliação audiológica.

No Brasil, Pádua e colaboradores (2005), em estudo desenvolvido a partir da implantação de um programa de TAN no Berçário de alto risco do HCFMUSP, verificaram que dentre os 1127 recémnascidos triados, 107 (9,5\%) apresentaram alterações em uma das avaliações. Destes, 23 casos foram encaminhados diretamente para ambulatório de diagnóstico da audição e $84(78,5 \%)$ casos foram encaminhados para reteste, sendo que apenas 32 $(34,4 \%)$ recém-nascidos compareceram.

Em outro estudo realizado no Brasil (Durante e col., 2004) com lactentes de alojamento conjunto, ob- 
teve-se taxa de adesão de 73,1\% dos que falharam.

Fernandes e Nozawa (2010) indicam outros serviços de TAN em maternidades no Brasil que, em seu primeiro ano de funcionamento, obtiveram os seguintes percentuais de não retorno para a conclusão da triagem auditiva: Itanhaém - 30\%, Maternidade do Hospital Universitário de São Paulo - 26,9\%, ou seja, taxas de adesão de 70\% e 73,1\%, respectivamente.

Assim, múltiplos aspectos parecem influenciar a adesão de lactentes a programas de triagem auditiva neonatal. Fernandes (2005) pesquisou os motivos para o não comparecimento do lactente ao reteste, apontando um conjunto de razões: baixa escolaridade dos pais, dificuldades financeiras para trazer a criança para a avaliação, confusão em relação aos diferentes agendamentos e encaminhamentos pósnatal, e o fato de a mãe possuir mais filhos e observar no cotidiano as reações do lactente aos sons. Sugere ainda que o desconhecimento das mães em relação ao direito de seus filhos à triagem e às repercussões da surdez no desenvolvimento da fala e da linguagem da criança corroboram para a não adesão.

Padua e colaboradores. (2005) elencam as seguintes possíveis razões do não comparecimento de lactentes para a triagem: sistema de marcação inadequado, falta de contato telefônico, dificuldade de transporte ao hospital e falta de comunicação entre a família do paciente e o médico em relação à importância do acompanhamento para a detecção de perdas auditivas tardias.

No estudo efetuado por Griz e colaboradores (2009), foram encontrados como fatores socioeconômicos e demográficos entre as mães que não completaram a triagem auditiva agendada: mães que tinham escolaridade inferior ao ensino médio, que eram provenientes de famílias de baixa renda e viviam em áreas rurais fora do município de Recife.

Estudo realizado por Ribeiro e Mitre (2004), no Hospital e Maternidade Vital Brasil, na cidade de Timóteo-MG, teve como objetivo avaliar o conhecimento das mães de recém-nascidos em relação à TAN. Foram entrevistadas cerca de 50 mães, entre elas 25 cujos filhos nasceram pelo Sistema Único de Saúde (SUS), e outras 25 cujos filhos tiveram cobertura de convênio ou particular. Verificou-se que apenas $22 \%$ das mães entrevistadas sabiam o que é a TAN e $78 \%$ nunca tiveram conhecimento.
Apesar de todas as mães entrevistadas acharem importante a realização da TAN, somente $6 \%$ delas autorizaram a realização do exame em seus lactentes. Segundo os autores do estudo, as usuárias que possuem convênio médico têm chance de saber o que é a TAN 6,47 vezes maior do que as usuárias que utilizam do SUS.

Olusanya e colaboradores (2004) discutem os desafios para Programas de Triagem Auditiva Neonatal para os países em desenvolvimento, apontando, entre outros fatores, o desafio do seguimento. Lembram eles de que a tarefa de completar o processo de triagem através do diagnóstico e da intervenção apropriada e no tempo adequado pode ser dura. A localização geográfica e as circunstâncias socioeconômicas dos pais desempenham um papel vital quanto a isso. Alguns pais simplesmente podem não estar interessados em continuar o processo após a triagem inicial.

Swanepoel e colaboradores (2007) deixaram que os pais de lactentes que falharam na TAN escolhessem se retornariam para o reteste no hospital onde fora realizada a triagem ou se buscariam um audiologista de sua escolha. Apenas 32\% retornam ao hospital e, com isso, não foi possível estabelecer as taxas de retorno. A partir disso, sugerem que os programas devem implementar o gerenciamento de dados e sistemas de seguimento que garantirão que as crianças sejam seguidas e os dados documentados. Isso é essencial para a provisão de serviços para as famílias e também para suprir dados compreensivos sobre a eficiência e a efetividade de programas e para o controle de qualidade.

Embora a adesão tenha sido quase sempre apontada como um fator que se relaciona ao usuário, não se pode negar que outros determinantes devem ser levados em conta, tais como: o contexto e a realidade em que os usuários vivem, a equipe que compõe o serviço de saúde e sua dinâmica de trabalho, os horários de atendimento oferecidos, o acolhimento, ou seja, diversos fatores "multirrelacionais" (Narciso e Paulilo, 2001).

Nesse sentido, podemos elencar alguns aspectos que explicariam a maior adesão na segunda etapa da avaliação em nosso estudo, como, por exemplo, a de que as mães passaram por orientações na triagem inicial, recebendo informações não somente sobre o processo da triagem, mas sobre as repercussões 
da perda auditiva no desenvolvimento infantil. Assim, ao receberem o resultado de que o lactente não passou, podem ter tido preocupação com relação à audição da criança o que as moveu a comparecer à segunda avaliação. Ou seja, sentiram-se responsáveis pela conclusão da avaliação, incluíram-se no processo e levaram a criança para realizar o exame. Algumas estratégias ainda podem ter corroborado para a maior adesão: o acolhimento das mães após o resultado 'falhou' da primeira avaliação, o acompanhamento contínuo até o retorno efetivo, buscando junto aos que faltavam recursos para minimizar as dificuldades para trazer a criança para a avaliação, dificuldades como o custo do transporte, o horário e os dias agendados, dentre outros.

\section{Conclusão}

A taxa de adesão obtida no Programa de Triagem Auditiva Neonatal no ano de 2007 foi a mais alta desde a implantação do programa, 79,34\% de retornos no reteste.

O aumento na taxa de adesão relativa ao período da pesquisa pode indicar algumas possíveis estratégias que contribuíram para maior participação no Programa: o trabalho de orientação às mães/ pais dos lactentes que vêm para a primeira etapa da triagem e o trabalho de acompanhamento pari passu das famílias dos lactentes que falharam na primeira avaliação, em relação às etapas seguintes do processo de triagem.

\section{Referências}

BRASIL. Ministério da Saúde. Portaria n. 2073 de 28 de setembro de 2004. Institui a Política Nacional de Atenção à Saúde Auditiva. Disponível em: <http:// dtr2001.saude.gov.br/sas/PORTARIAS/Port2004/ GM/GM-2073.htm>. Acesso em: 29 jan. 2009.

CAMPINAS. (Prefeitura). Decreto n. 14.640 de 16 de Fevereiro de 2004. Regulamenta a lei $n^{\circ}$. 10.759, de 20 de Dezembro de 2000. "Dispõe sobre a obrigatoriedade da realização de exames que detectem a surdez ou alterações correlatas, nas maternidades e estabelecimentos hospitalares congêneres do município de Campinas". Disponível: <http://2009.campinas.sp.gov.br/ bibjuri/dec1464o.htm>. Acesso em: 14 abr. 2007.
COMITÊ BRASILEIRO SOBRE PERDAS AUDITIVAS NA INFÂNCIA. RECOMENDAÇÃO o1/99 do Comitê Brasileiro sobre Perdas Auditivas na Infância. Jornal do Conselho Federal de Fonoaudiologia, 200o. p. 3-7.

DE CAPUA. B. et al. Universal neonatal hearing screening: the Siena (Italy) experience on 19700 newborns. Early Human Development, Amsterdam, v. 83, n. 9, p. 601-6o6, sept. 2007.

DURANTE. A. S. et al. A implementação de programa de triagem auditiva neonatal universal em um hospital universitário brasileiro. Pediatria (São Paulo), v. 26, n. 2, p. 78-84, 2004.

FERNANDES, J. C. Estudo da efetividade de um programa de Triagem Auditiva Neonatal Universal. Dissertação (Mestrado em Enfermagem) - Faculdade de Ciências Médicas, Universidade Estadual de Campinas, Campinas, 2005.

FERNANDES, J. C.; NOZAWA, M. R. Estudo da efetividade de um Programa de Triagem Auditiva Neonatal Universal, Ciência \& Saúde Coletiva, Rio de Janeiro, v. 15, n. 2, p. 353-361, mar. 2010. Disponível em: <http://www.scielosp.org/scielo. php?pid=S1413-81232010000200010\&script=sci arttext>. Acesso em: 17 jan. 2009.

FINITZO, T.; ALBRIGHT, K.; O'NEAL, J. The Newborn with hearing loss: detection in the Nursery. Pediatrics, Springfield, v. 102, n. 6, p. 1452-146o, dec. 1998.

FRANÇOZO, M. F. C. et al. Improvement of return rates in a neonatal hearing screening program: the contribution of Social Work. Social work in health care, New York, v. 44, n. 3, p. 179-19o, 2007. GATTO, C. L.; TOCHETTO, T. M. Deficiência Auditiva Infantil: implicações e soluções. Revista CEFAC, São Paulo, v. 9, n. 1, p. 110-15, jan.-mar. 2007.

GRIZ, S. et al. Newborn hearing screening: an outpatient model. International Journal of Pediatric Otorhinolaryngology, Amsterdam, v. 73, n. 1, p. 1-7, jan. 2009.

ISAACSON, G. Universal Newborn Hearing screening in an inner-city, managed care environment. The Laryngoscope, St. Louis, Mo, v. 110, n. 6, p. 881-894, june 2000. 
JOINT COMMITTEE ON INFANT HEARING et

al. Year 200o. Position statement: principles and guidelines for early hearing detection and intervention programs. Joint Committee on Infant Hearing, American Academy of Audiology, American Academy of Pediatrics, American Speech-Language-Hearing Association, and Directors of Speech and Hearing Programs in State Health and Welfare Agencies. Pediatrics, Springfield, v. 106, n. 4, p. 798-817, oct. 2000.

\section{JOINT COMMITTEE ON INFANT HEARING.}

Year 2007. Position Statement: principles and guidelines for early Hearing Detection and Intervention Programs. Pediatrics, Springfield, v. 120, n. 4, p. 898-921, oct. 2007. Disponível em: <http://pediatrics.aappublications.org/cgi/ content/full/120/4/898>. Acesso em: 23 fev. 2009.

LIMA, G. M. L. Análise da triagem auditiva por audiometria automática de tronco encefálico de recém-nascidos internados em unidade de cuidados intensivos e intermediários. Dissertação (Mestrado em Saúde da Criança e do Adolescente) - Faculdade de Ciências Medicas, Universidade Estadual de Campinas, Campinas, 2004.

LIU, C. et al. Evaluating loss to follow-up in newborn hearing screening in Massachusetts. Pediatrics, Springfield, v. 121, n. 2, p. 335-343, feb. 2008. Disponível em: <http://pediatrics. aappublications.org/cgi/content/abstract/121/2/ e335>. Acesso em: 26 maio 2009.

MEHL, A. L.; THOMSON, V. Newborn hearing screening: the great omission. Pediatrics, Springfield, v. 101, n. 1, jan. 1998. Disponível em: <http://pediatrics.aappublications.org/cgi/ content/abstract/101/1/e4?maxtoshow=\&hits $=10 \&$ RESULTFORMAT $=\&$ fulltext=Newborn+hearing $+\mathrm{sC}$ reening\%3A+the+great+omission\&searchid $=1 \& F I R$ STINDEX $=0 \&$ sortspec $=$ relevance $\&$ resourcetype $=\mathrm{H}$ WCIT $>$. Acesso em: 17 jan. 2009.

NARCISO, A. M. S.; PAULILO, M. A. S. Adesão e AIDS: alguns fatores intervenientes. Serviço Social em Revista, Londrina, v. 4, n. 1, p. 27-43, jul.dez. 2001. Disponível em: <http://www.ssrevista. uel.br/nıv4.pdf $>$. Acesso em: 17 jan. 2009.
OLUSANYA, B. O.; LUXON, L. M.; WIRZ, S. L. Benefits and challenges of newborn hearing screening for developing countries. International Journal of Pediatric Otorhinolaryngology, Amsterdam, v. 68, n. 3, p. 287-305, mar. 2004. OZCEBE, E.; SEVIN, S.; BELGIN, E. The ages of suspicion, identification, amplification and intervention in children with hearing loss. International Journal of Pediatric Otorhinolaryngology, Amsterdam, v. 69, n. 8, p. 1081-1087, aug. 2005.

PÁDUA, F. G. C. et al. Triagem Auditiva Neonatal: um desafio para sua implantação. Arquivos Internacionais de Otorrinolaringologia, São Paulo, v. 9, n. 3, p. 190-194, 2005.

RIBEIRO, F. G.; MITRE, E. I. Avaliação do Conhecimento sobre triagem auditiva Neonatal de pacientes no pós-parto imediato. Revista CEFAC, São Paulo, v. 6, n. 3, p. 294-299, 2004.

ROSSI, T. R. F. et al. Triagem Auditiva Neonatal: acompanhamento de bebês que falharam na primeira Avaliação. In: CONGRESSO BRASILEIRO DE EDUCAÇÃO ESPECIAL, 1., ENCONTRO DA ASSOCIAÇÃO BRASILEIRA DE PESQUISADORES EM EDUCAÇÃO ESPECIAL, 1.,2003, São Carlos. Anais... São Carlos, SP, p.335-336.

SWANEPOEL, D. et al. Newborn hearing screening in a South African private health care hospital. International Journal of Pediatric Otorhinolaryngology, Amsterdam, v. 71, n. 6, p. 881-887, june 2007.

UUS, K.; BAMFORD, J. Effectiveness of populationbased newborn hearing screening in England: ages of intervention and profile of cases. Pediatrics, Springfield, v. 117, n. 5, p. 887-893, may 2006. Disponível em: <http://pediatrics. aappublications.org/cgi/content/full/117/5/e887>. Acesso em: 26 maio 2009.

YOSHINAGA-ITANO, C. et al. Language of early and later-identified children with hearing loss. Pediatrics, Springfield, v. 102, n. 5, p. 1161-1171, nov. 1998.

Recebido em: 05/10/2009 Reapresentado em: 01/09/2010 Aprovado em: 06/09/2010 